\title{
The Constituents and Potential Targets of the Extracellular Matrix: Implications for Carcinogenesis and Cancer Treatment
}

\author{
Weber CE, Driver J, Franzen CA, Mascarenhas JB, Mi Z, Gupta GN, Wai PY and Kuo PC*
}

Department of Surgery, Loyola University, Maywood, Ireland

\begin{abstract}
The dense extracellular matrix consists of a multitude of proteins with important implications in tumorogenesis that extend beyond the maintenance of tissue integrity. Several of the main macromolecular constituents- proteoglycans, collagens, integrins, and syndecans will be discussed in this review, with particular attention to their roles in tumor initiation, invasion, angiogenesis, and metastasis. In addition, a brief synopsis of the role of enzymes that remodel the extracellular matrix will be provided. Finally, specific examples of targeted molecular therapies: anti-integrin agents, MMP inhibitors, and hyaluronidase will be discussed.
\end{abstract}

Keywords: Extracellular matrix; Proteoglycans; Integrins; Syndecans; Matrix metalloproteinases; Hyaluronidase

Abbreviations: ECM- Extracellular Matrix; BM- Basement Membrane; PG- Proteoglycan; HSPG- Heparan Sulfate Proteoglycan; GAG- Glycosaminoglycan; MMP- Matrix Metalloproteinase; ADAMA Disintegrin And Metalloproteinase; TIMP- Tissue Inhibitor of Metalloproteinases; VEGF- vascular endothelial growth factor; HFG- Hepatocyte Growth Factor; FGF2- Fibroblast Growth Factor 2; TGF $\beta$ - Transforming Growth Factor $\beta$; PDGF- Platelet-Derived Growth Factor; IGF- Insulin-Like Growth Factor; EMT- EpithelialMesenchymal Transition

\section{Introduction}

The biology of cancer is intricate and multifaceted. Much is known about genetic and epigenetic changes that transform normal cells into an aggressive malignant phenotype. However, cancer cells exist in a complex microenvironment, dependant on feedback and paracrine signaling from other tumor cells and from stromal cells such as fibroblasts, immune cells, and pericytes. In addition, the dynamic, reciprocal network of proteoglycans, polysaccharides, glycoproteins, growth factors, and other soluble molecules that constitute the Extracellular Matrix (ECM) also has a profound influence on carcinogenesis and metastasis. These main constituents of the ECM as well as their roles in tumorogenesis, invasion, angiogenesis, and metastasis will be discussed in this review. Specific ECM targeted molecular anti-cancer therapies currently under investigation will be highlighted.

\section{Extracellular Matrix}

Extracellular matrix (ECM) is the dense, often rigid, non-cellular environment that is in direct physical contact with cancer cells during all stages of tumorogenesis and metastasis. The components of ECM include: proteoglycans, glycoproteins, polysaccharides, water, and soluble factors [1,2]. According to Hynes and Naba, the "core matrisome" of proteins in the ECM is vast, numbering around 300 [3]. This milieu of macromolecules constantly undergoes posttranslational modification, degradation, and remodeling by enzymes such as Matrix Metalloproteinases (MMPs). Typically, the term ECM is used to describe the interstitial matrix, or the space between cells. A more specialized version of ECM is located basolaterally to epithelial and endothelial cells, and is termed the Basement Membrane (BM) [4]. Besides forming the architectural scaffolding and imparting structural integrity to tissues, the ECM plays an important role in biochemical and mechanical signaling in the Tumor Microenvironment (TME). These cues modulate many aspects of carcinogenesis, from tumor formation to tumor migration and invasion to distant metastasis [1,2] Communication between the ECM and cancer cells occurs directly through cell-ECM adhesion molecules such as integrins and syndecans and also indirectly through ECM bound growth factors and transmitted mechanical forces. The main macromolecules constituting the ECM as well as the adhesion molecules connecting cancer cells to the ECM will be described in the following sections.

\section{Macromolecules of the ECM}

\section{Proteoglycans}

Proteoglycans (PGs) are composed of a core protein covalently attached via serine residues to long unbranched polysaccharides made up of repeating disaccharide units- glycosaminoglycan chains (GAGs). These GAGs are highly sulfated imparting a net negative charge that attracts water as well as cations and creates a hydrated gel-like environment that permits resistance to compressive forces and sequesters soluble growth factors. There are five known GAGs: heparan sulfate, chondroitin sulfate, dermatan sulfate, keratin sulfate, and hyaluronan. All exist bound to a protein core except hyaluronan, also known as hyaluronic acid (HA), which is released into the ECM independent of the Golgi apparatus and exists bound to the matrix and to the cell surface via CD44 receptors. PGs can be divided into four main families: glypicans (six heparan sulfate PGs covalently anchored to the cell surface via GPI-glycosylphosphatidylinositol), syndecans (four primarily heparan sulfate or chondroitin/dermatan sulfate transmembrane PGs), lecticans (four chondroitin or keratin sulfate soluble PGs located extracellularly), and SLRPs- small leucine-

*Corresponding author: Paul C. Kuo, Loyola University Medical Center, Department of Surgery, 2160 S First Ave, Maywood, IL 60153, Ireland, Tel: 708327-2705; Fax: 708-327-2852; E-mail: pkuo@lumc.edu

Received April 09, 2013; Accepted May 25, 2013; Published June 05, 2013

Citation: Weber CE, Driver J, Franzen CA, Mascarenhas JB, Mi Z, et al. (2013) The Constituents and Potential Targets of the Extracellular Matrix: Implications for Carcinogenesis and Cancer Treatment. J Carcinogene Mutagene S13: 006. doi:10.4172/2157-2518.S13-006

Copyright: (c) 2013 Weber CE, et al. This is an open-access article distributed under the terms of the Creative Commons Attribution License, which permits unrestricted use, distribution, and reproduction in any medium, provided the original author and source are credited. 
rich repeat proteoglycans (six chondroitin/dermatan sulfate or keratin sulfate PGs also located extracellularly). There are many PGs that are not members of a specific family, for example, CD44, thrombomodulin, perlecan, endocan, and type IX collagen among others [5,6].

In tumorogenesis, much attention has been paid to the ubiquitous heparan sulfate proteoglycans (HSPGs) [7]. The repeating disaccharide units in HSPGs are composed of glucuronic acid and $\mathrm{N}$-acetylglucosamine. Complexity is conferred to these molecules by epimerization, sulfation, and de-acetylation [6]. The main types of interstitial matrix HSPGs are glypicans and syndecans, while examples of BM HSPGs are collagen type XVIII, perlecan, and agrin $[1,5]$. In addition to contributing to tissue integrity via their interactions with collagens and other glycoproteins such as fibronectin and laminin in the ECM, HSPGs also mediate cellular uptake of growth factors and other soluble ligands and function as co-receptors for multiple ligands. They mediate important cell signaling pathways involved in cellular growth, proliferation, and migration [7]. Multiple studies also implicate HSPGs as extracellular reservoirs for growth factors, chemokines, morphogens, and cytokines. In this bound form, these soluble factors are inactive and unable to influence their target cells. However, cleavage of HS by heparanase (an endoglucuronidase) results in the release of HS bound factors such as Vascular Endothelial Growth Factor (VEGF), Hepatocyte Growth Factor (HGF), Fibroblast Growth Factor 2 (FGF2), Transforming Growth Factor $\beta$ (TGF $\beta$ ), and Platelet-Derived Growth Factor (PDGF), which can potentiate tumor cell growth and invasion as well as angiogenesis and metastasis $[6,8]$. Studies have shown that in melanoma, for example, the release of HS by heparanase promotes melanoma tumorogenesis and local angiogenesis [8]. Elevated heparanase levels have been detected in many other cancers, and have been correlated with invasion and metastasis [9]. However, non-enzymatic functions of heparanases in carcinogenesis have been described [6].

Another class of enzymes that act on HSPGs, sulfatases (Sulfs), is also frequently dysregulated in cancer. These enzymes modify the sulfation status of HSPGs by selectively removing a sulfate group from the 6-O position, resulting in altered binding of ligands. While classic theory holds that Sulf- 1 is a tumor suppressor and Sulf- 2 is pro-tumor, Sulf- 1 and Sulf- 2 levels have been shown to be both up-regulated and down-regulated in human cancers $[10,11]$. Thus, the role of HSPGs in carcinogenesis is highly complex.

\section{Collagens}

Collagens are a group of 28 fibrous proteins that exist as supramolecular complexes of triple-stranded $\alpha$-helices. Post-translationally, hydroxylation occurs at proline and lysine residues, while glycosylation occurs at hydroxylysine residues [12]. These fibrils and networks are further organized into sheets and cables by fibroblasts and provides tensile strength to the ECM and BM [1]. The main collagen components of the interstitial matrix are types I and III, whereas collagen type IV is abundant in the BM [4]. Collagens regulate cell adhesion to the ECM by binding to cell surface adhesion molecules including integrins, syndecans, and other PGs. The ECM of cancer is characterized by a dense, rigid, parallel orientation of collagen fibers that promotes epithelial tumor cell migration [12]. Stiffness of the microenvironment is created in part by covalent crosslinking of collagen fibers, facilitated by lysyl oxidase enzymes (LOX and LOXL1-4). These copper dependent enzymes are upregulated in many cancers, especially under hypoxic conditions, and their overexpression results in a rigid ECM that is tumorogenic and supportive of invasion and metastasis. The LOX dependent collagen crosslinking up-regulates the formation of integrin focal adhesion complexes stimulating downstream signaling, further demonstrating the complex dynamic interactions that occur during tumor development and progression [13]. Studies have shown that upregulation of LOX occurs in more invasive and aggressive cancers, and correlates with poor prognosis $[12,14,15]$.

Desmoplasia, or fibrotic reaction, driven primarily by stromal fibroblasts, either resident or recruited, represents an important step in tumorogenesis. ECM proteins, including collagens, are laid down in an extensive, dense network and facilitate directional migration of tumor cells through this collagen "highway" $[12,13]$. Using a pancreatic cancer model, one study reported that stromal fibroblasts expressing the protease- Fibroblast Activation Protein (FAP) promote the formation of an organized and parallel network of collagen that enhances velocity and directionality of invading tumor cells in a $\beta 1$-integrin/FAK dependent fashion [16]. In addition, the mechanical force of this stiff matrix is sensed and transmitted via integrin receptors, resulting in increased epithelial tumor cell proliferation $[12,13]$.

Collagen fibril formation is also greatly influenced by the glycoprotein fibronectin, which can bind directly to collagen fibers $[12,17]$. In many cancers, fibronectin dimer production by stromal fibroblasts is upregulated. These globular dimers associate with $\alpha 5 \beta 1$ integrins on the cell surface and the protein is stretched and elongated into a more linear form, revealing cryptic binding sites on fibronectin that when exposed may further contribute to fibril strength and rigidity [18]. Thus, the relationship between collagen fibril formation and fibronectin fibrillogenesis is reciprocal in nature and involves integrin receptors [17].

\section{ECM-Cell Adhesion Molecules}

\section{Integrins}

Integrins are a class of 24 distinct heterodimeric cell-surface glycoproteins composed of $\alpha$ and $\beta$ subunits. Multiple classifications exist for integrins, but generally revolve around binding specificities. They are characterized by the presence of multiple binding and activation sites and are known to interact with a multitude of cell adhesion molecules, other proteins of the ECM, and growth factors [1921]. This complex integrin network, or "integrin adhesome" consists of 156 linked proteins and lipids characterized by 690 interactions (379 binding, 213 activation, and 98 inhibitory) [22].

Integrins do not possess intrinsic enzymatic activity, but they facilitate cell adhesion to the ECM and mediate "inside-out" signal transduction between the ECM and cancer cells through nonenzymatic mechanisms. When bound to other proteins or growth factors in the ECM, integrins undergo receptor clustering and conformational changes that expose effector-binding sites. The result is the activation of cytoplasmic kinases, such as the phosphorylation of Focal Adhesion Kinase (FAK), which results in the transmission of signals to the cell's nucleus. These transmitted signals modulate cell migration, proliferation, and metastasis [19-21]. In addition, integrin signaling pathways regulate polymerization and de-polymerization of the actin cytoskeleton at the leading edge of cells, necessary for cell spreading on the ECM [20].

A major class of integrins up-regulated in the ECM of many tumors is arginine-glycine-aspartate (RGD) binding integrins. Examples of this family implicated in cancer and angiogenesis include: $\alpha v \beta 3, \alpha v \beta 1$, $\alpha v \beta 5$, and $\alpha 5 \beta 1$. Tumor cells deposit glycoproteins containing the RGD sequence (ex. fibronectin and vitronectin) into the ECM during 
the initiation of tumorogenesis. These protein-protein interactions promote tumor formation and migration $[23,24]$.

\section{Syndecans}

Syndecans are a class of transmembrane HSPGs that are crucial for cell-ECM adhesion and paradoxically, also cell migration. Four syndecans (syndecan-1, $-2,-3$, and -4 ) have been characterized, but syndecan-1 is currently the most described in cancer literature [25]. It has been shown that basolaterally expressed syndecan-1 functions to anchor epithelial cells by connecting the ECM to the actin cytoskeleton via its associations with collagens, fibronectin, and thrombospondin. Investigators have also demonstrated that syndecan-1 associates with integrins and stabilizes focal adhesion complexes. The expression of cell surface syndecan-1 on epithelial cells promotes cell adhesion and prevents cell locomotion and migration [26]. Somewhat paradoxically, the expression of syndecan- 1 on stromal fibroblasts actually promotes tumor cell migration. In models of breast cancer, it has been shown that syndecan-1 positive stromal fibroblasts orchestrate the formation of an organized ECM with collagen fibers in a parallel orientation, which promotes directional migration and invasion of cancer cells [25].

Syndecan-1 is also implicated in cancer progression via another mechanism. The syndecan proteins have an extracellular domain (ectodomain) that can be cleaved or shed by sheddases, which are membrane-bound enzymes of the MMP or A Disintegrin And Metalloproteinase (ADAM) families [26,27]. For example, one study reported that in vitro, membrane type matrix metalloproteinase-1 (MT1-MMP also termed MMP-14), cleaves a Gly245-Leu246 peptide bond that results in the shedding of syndecan- 1 and the stimulation of cell migration [28]. When this ectodomain is shed, this soluble protein promotes migration and angiogenesis. In fact, high levels of shed syndecan-1 in patients' serum of many cancers have been correlated with a poor prognosis. For example, in multiple myeloma, heparanase expression in the bone marrow, which results in the shedding of syndecan-1, is a bad prognostic factor [29]. Another study suggested that levels of shed syndecan-1 in the serum might even be an independent prognostic marker in multiple myeloma [30]. One proposed mechanism of how shed syndecan-1 participates in carcinogenesis is through its binding to angiogenic growth factors, VEGF and FGF-2. Receptors such as VEGFR and integrins on endothelial cells can recognize these growth factor-syndecan-1 complexes and initiate a process of endothelial budding and invasion eventually leading to neovasculature formation [26].

\section{ECM Remodeling and Degradation}

\section{MMPs/TIMPs}

The ECM of tumors is continually undergoing degradation and remodeling by proteases. The primary proteases involved are members of the MMP family, zinc dependent endopeptidases. Currently over 20 members have been identified and are divided into four main groups: collagenases, gelatinases, membrane type, and stromelysins. Other MMPs such as matrilysin (MMP-7) do not readily fit into any of the above groups. Both tumor and stromal cells secrete mMPs as inactive zymogens in the form of pro-MMPs and are activated in the ECM by other proteases or by already active MMPs. The mechanisms and functions of MMPs in tumorogenesis, angiogenesis, and metastasis are diverse [31-33].

Normal tissue homeostasis is maintained by tight control of MMP activity, however in tumorogenesis, cancer cells exploit the function of these enzymes to promote an invasive and metastatic phenotype
[31-33]. In addition, when normal epithelial cells lose their connection to the ECM they undergo anoikis (programmed cell death), which is triggered by detachment. Cancer cells, on the other hand, have evolved mechanisms to avoid anoikis, allowing them to survive even after MMPs disrupt their attachments to the ECM and to each other [34].

Two tumorogenic MMPs that have been extensively studied in cancer are the gelatinases: MMP-2 and MMP-9. These MMPs and others cleave ECM protein-growth factor complexes and release bound factors, such as VEGF, TGF $\beta$, and IGFs, which can then bind to growth factor receptors and promote tumor proliferation and angiogenesis $[35,36]$. MMP-9 has also been shown to interact with the av $\beta 3$ integrin receptor in breast cancer [31]. In addition, MMPs are involved in the disruption of epithelial and endothelial cell basement membranes, which promote cell migration and angiogenesis [31-33]. For example, cleavage of type IV collagen and laminin in the BM uncovers cryptic binding sites for growth factors and other proteins that promote tumor cell growth, migration, and angiogenesis. [37] On the other hand, cleaved products from type IV collagen are also known to be antiangiogenic [38].

Another important step of tumorogenesis promoted by MMPs as well as ADAMs is the degradation of cell-cell adhesion molecules such as cadherins, a family of calcium binding transmembrane glycoproteins that form adherens junctions between epithelial cells. The downregulation of E-cadherin, a process regulated by many factors in addition to MMPs and ADAMs, results in the translocation of $\beta$-catenins to the nucleus and the polymerization of the actin cytoskeleton into stress fibers. A more motile mesenchymal-like cell now characterizes the invasive and metastatic phenotype [31]. This phenomenon termed the epithelial-mesenchymal transition (EMT) is mediated largely by TGF $\beta$. Other proteins such as $\mathrm{N}$-cadherin, vimentin, tenascin- $\mathrm{C}$, and fibronectin are up-regulated in the mesenchymal phenotype. Further details of EMT are beyond the scope of the discussion in this review [39-41].

The proteolytic degradation and remodeling of MMPs is inhibited by the activity of four tissue inhibitors of metalloproteinases (TIMP14). The N-terminus of TIMPs binds to the catalytic domain of MMPs and inhibits their activity. In tumorogenesis, the balance of MMPs and TIMPs is altered, and decreased expression or down-regulation of TIMPs promotes tumor metastasis as well as angiogenesis [31].

As mentioned above, one class of MMPs is the membrane type MMPs that are expressed on the cell surface. MMP-14 or MT1-MMP is a well-studied member of this class. This isoform functions to degrade collagen types I, II, and III as well as fibronectin, laminins, vitronectin, and aggrecan. It has been shown to be present on the motile edge of tumors and serves to cleave the hyaluronan receptor CD44, promoting cell migration [42]. In fact, elevated levels of soluble CD44 have been detected in the plasma of patients with metastatic cancer [43]. In addition, MT1-MMP, in conjunction with TIMP-2 recruits and activates pro-MMP-2. This paradoxical pro-tumor effect of TIMP-2 is attributed to its C-terminal domain $[31,33,44,45]$.

\section{ECM-Targeted Molecular Anti-Cancer Agents}

\section{Anti-Integrin Drugs}

Goodman and Picard, in their review on anti-integrin agents, highlighted the properties of integrins, specifically their cell-surface location and their sensitivity to blockade that make them attractive therapeutic targets. Approximately 15 anti-integrin agents were currently in active clinical trials as of 2012 [23]. Cilengitide, the only 
anti-integrin drug in a Phase III trial- CENTRIC (clinicaltrials.gov, NCT00689221), is a small molecule drug with an RGD sequence that inhibits both $\alpha v \beta 3$ and $v \beta 5$ integrin receptors. This drug has shown favorable safety profiles in earlier trials, however, in February of 2013, Merck reported that the study failed to meet its primary endpoint of a survival advantage in the group receiving cilengitide in conjunction with standard chemoradiotherapy compared to the group receiving only standard chemoradiotherapy in patients with glioblastoma multiforme [46]. The full results of the study are not yet available, but will reportedly be available in June 2013. Currently, other Phase I and II trials using cilengitide as a therapeutic for multiple other cancers are in various stages of development [47-50]. (See also: NCT01276496, NCT01118676, NCT00705016, NCT00077155)

Other integrin inhibitors demonstrating potentially promising results in Phase I and II clinical trials include: 1. Etaracizumab (MedImmune), a monoclonal antibody to av $\beta 3$ integrin; 2 . Volociximab (PDL BioPharma/ Biogen Idec), a monoclonal antibody to $\alpha 5 \beta 1 ; 3$. ATN-161 (Tactic Pharma), an oligopeptide that inhibits av $\beta 3$, $\alpha 5 \beta 1$, and $\alpha v \beta 5$ in an RGD-independent manner; 4. Intetumumab (Janssen Biotech), a monoclonal antibody to av integrin; and 5. DI17E6 (Merck $\mathrm{KGaA} / \mathrm{EMD}$ Serono), also a monoclonal antibody to av integrin.

Etaracizumab (MEDI-522) is currently under investigation in melanoma (NCT00066196) and advanced prostate (NCT00072930) and colorectal cancer (NCT00027729). A randomized phase II openlabel trial comparing the combination of etaracizumab and dacarbazine versus dacarbazine alone in patients with metastatic melanoma, however, showed no benefit in time to progression or progression free survival with the addition of etaracizumab [51]. Volociximab is in various stages of safety and efficacy trials in melanoma (NCT00099970), non-small cell lung (NCT00654758), renal (NCT00100685), ovarian (NCT00516841), and pancreatic cancer (NCT00401570). However, a phase II study showed progression of disease in 13 of 14 patients receiving monotherapy volociximab for platinum-resistant ovarian or peritoneal cancer [52].

Preliminary data from a phase I trial of ATN-161 in 26 patients with solid tumors showed the drug was well tolerated [53], and a current trial is underway in malignant gliomas (NCT00352313). Also, according to Tactic Pharma, phase II trials in head and neck cancer as well as glioblastoma multiforme are in the planning stages. A randomized phase II trial (NCT00246012) in metastatic melanoma patients of intetumumab administered alone or in combination with dacarbazine showed no significant difference in progression free survival [54]. Another study, also phase II (NCT00537381), however, showed inferior progression free survival in patients who received intetumumab in combination with docetaxel and prednisone compared to docetaxel and prednisone alone in patients with metastatic castration-resistant prostate cancer [55]. Finally, as of April 2012, a phase II study (PERSEUS, NCT01360840) of DI17E6 (also referred to as EMD 525797) had enrolled 106 patients with metastatic castrateresistant prostate cancer. Results of that study are not yet available [56].

Anti-integrin therapy is still in its infancy, and there are still many unanswered questions regarding the therapeutic potential for drugs like Cilengitide. The reasons for failure of many of the abovementioned studies are likely not a simple answer. There is concern about target specificity, as normal cells express integrin receptors as well. In addition, preclinical animal data has raised concerns that antiintegrin agents might actually increase tumor growth and angiogenesis [57]. Hersey at al, also point out that determining the optimal dose of anti-integrins is crucial, and hypothesize that higher doses of etaracizumab $(>10 \mathrm{mg} / \mathrm{kg})$ might have produced a survival difference in their study [51]. Finally, the majority of these trials have been conducted in advanced stage cancers. Perhaps anti-integrin therapy might prove more beneficial if given early on in carcinogenesis. Future studies using these targeted molecular therapies will hopefully answer these questions.

\section{MMP Inhibitors}

Unfortunately, despite the fact that MMPs are up-regulated in almost all human tumors and play a vital role in tumor progression via matrix remodeling and digestion of cell adhesion molecules, the results from clinical trials looking at MMP inhibitors have been largely disappointing. Early studies showed that with prolonged usage, MMP inhibitors resulted in significant musculoskeletal pain and inflammation. This toxicity was reduced by the development of drugs with diminished activity against sheddases. However, Phase III clinical trials have failed to show therapeutic benefit or improved survival [32,58-60]. A phase III trial (NCT00004199) comparing prinomastat in combination with gemcitabine-cisplatin in patients with non-small cell lung cancer was closed early due to lack of efficacy [58]. A randomized phase III trial of tanomastat was conducted after administration of chemotherapy to patients with ovarian cancer, but failed to demonstrate any prolongation in progression free survival [59]. Marimastat, administered after chemotherapy in a phase III trial (NCT00003010) to patients with stable metastatic breast cancer, also failed to prolong progression free survival [60].

These results were especially discouraging as preliminary animal research using MMP inhibitors showed promising results. Coussens et al. points out, however, that when MMP inhibitors were used in animal models of cancer they were generally given early on in tumor formation, whereas in human studies the majority of patients had advanced stage or even metastatic disease [32]. Thus, MMP inhibitors may actually be useful in treating human cancer if given earlier on in disease progression, but this remains to be proven.

\section{Enhancing drug delivery through the ECM}

The desmoplastic reaction that results in a dense and stiff ECM not only promotes tumor progression by creating cell-ECM interactions but also provides chemoresistance to the developing tumor. Intratumoral vasculature is leaky and the lack of functional lymphatics contributes to this chemoresistance by raising the interstitial fluid pressure [12]. The presence of elevated amounts of HA in most cancers, a polysaccharide that attracts water, expands the matrix volume, and increases viscosity, further increases interstitial fluid pressure, creating issues with hematogenous chemotherapeutic drug delivery. It is also thought that the anionic nature of HA prevents adequate tumor penetration by chemotherapeutic agents [61]. Finally, it is likely that collagen fibers bind to and sequester drugs, further preventing their action in the tumor microenvironment [12].

Several studies carried out in animals have shown that there is a reduction in tumor volume with inhibition or depletion of stromal components, such as fibroblasts, that are primarily responsible for the fibrotic reaction in the ECM $[62,63]$. Others have demonstrated that inhibition of signaling pathways that potentiate stromal desmoplasia transiently increases the delivery of chemotherapeutic agents [64]. A temporary reduction in interstitial fluid pressure and an associated increase in delivery of antibodies were seen with collagenase and hyaluronidase in an osteosarcoma xenograft model [65-67].

Despite many promising animal models, enzymatic modification 


\begin{tabular}{|c|c|c|c|}
\hline Constituent & Classes/Families & Example Members & Proposed roles in cancer \\
\hline \multirow[t]{5}{*}{ Proteoglycans } & Glypicans & Glypican 1-6 & cell-ECM adhesion, proliferation \\
\hline & Syndecans & Syndecan 1-4 & $\begin{array}{l}\text { Cell surface- cell-ECM adhesion, sequestration of growth factors Shed } \\
\text { ectodomain- invasion, migration, angiogenesis }\end{array}$ \\
\hline & Lecticans & Aggrecan, Versican, Brevican, Neurocan & structural integrity, aggregation \\
\hline & SLRPS & $\begin{array}{l}\text { Decorin, Biglycan, Fibromodulin, Podocan, } \\
\text { Keratocan, and others }\end{array}$ & $\begin{array}{l}\text { cell-ECM adhesion, fibrillogenesis of ECM proteins, growth factor sequestration } \\
\text { and signaling, cell proliferation, migration, metastasis }\end{array}$ \\
\hline & Others & $\begin{array}{l}\text { CD44, Perlecan, Type IX collagen, Endocan, } \\
\text { and others }\end{array}$ & $\begin{array}{l}\text { cell surface co-receptors for growth factor signaling, migration, structural } \\
\text { integrity, angiogenesis }\end{array}$ \\
\hline \multirow[t]{2}{*}{ Collagens } & & Type I, III & $\begin{array}{l}\text { interstitial matrix, cell-ECM adhesion, facilitate directional migration, role in } \\
\text { interstitial pressure }\end{array}$ \\
\hline & & Type IV, XVIII & basement membrane matrix \\
\hline \multirow[t]{4}{*}{ Integrins } & RGD binding & $\alpha 5 \beta 1, \alpha v \beta 3, \alpha v \beta 5, \alpha v \beta 1, \alpha \vee \beta 6, \alpha v \beta 8$ & $\begin{array}{l}\text { cell-ECM attachment and "inside-out" signaling, migration, invasion, metastasis, } \\
\text { EMT }\end{array}$ \\
\hline & a4 family & $\alpha 9 \beta 1$ & cel-ECM adhesion, tumor growth, migration, invasion, angiogenesis, EMT \\
\hline & $\begin{array}{l}\text { I domain: collagen } \\
\text { binding }\end{array}$ & $\alpha 1 \beta 1, \alpha 2 \beta 1, \alpha 11 \beta 1$ & collagen fibril formation and polymerization \\
\hline & LN binding & $\alpha 6 \beta 4$ & migration, invasion, metastasis \\
\hline
\end{tabular}

Table 1: Specific examples of these ECM constituents and their reported roles in cancer.

of the ECM to improve drug delivery has been plagued with issues in human studies. For example, early studies using bovine hyaluronidase resulted in a high number of allergic and anaphylactic reactions. In addition, use of collagenases has been limited by its lack of specificity [61]. Currently, though, recombinant human hyaluronidase, Hyelen ${ }^{\circledR}$, is FDA approved for subcutaneous hydration and to enhance delivery of injectable drugs. In cancer research, Halozyme Therapeutics has developed a subcutaneous form that is under investigation, as Chemophase in superficial bladder cancer in early phase studies (NCT00782587, NCT00318643) in conjunction with Mitomycin $\mathrm{C}$ and as PEGPH20 in combination with gemcitabine for stage IV pancreatic cancer (NCT01453153). It has been shown that the addition of PEGPH20 to gemcitabine depletes HA, normalizes interstitial fluid pressure, increases tumor vasculature, decreases tumor size, decreases metastatic burden, and prolongs survival in a murine spontaneous pancreatic cancer model [68]. The hope is that the addition of PEGPH20 to chemotherapeutics will have the same positive results in human trials.

Additionally, Hoffman LaRoche has been granted a license by Halozyme to combine the recombinant human hyaluronidase with trastuzumab in the form of a subcutaneous injectable Herceptin ${ }^{\circledR}$. A recently resulted phase III trial (NCT00950300), the HannaH study, demonstrated non-inferiority of the subcutaneous Herceptin ${ }^{\circledR}$ compared to the traditional IV Herceptin ${ }^{\circledR}$ in female patients with early stage HER2 positive breast cancer [69-70]. Two more trials, PrefHer (NCT01401166) and SafeHer (NCT01566721) are actively recruiting participants to analyze patient preference as well as drug delivery devices for subcutaneous Herceptin ${ }^{\circledR}$.

\section{Conclusion}

The ECM, or the dense non-cellular component of tumors, is an important contributor to tumorogenesis and metastasis. Macromolecules such as proteoglycans, collagens, integrins, and syndecans, originally thought to function primarily in structural support have been found to participate in numerous diverse ECM-cell signaling pathways that promote tumor migration, invasion, and metastasis as well as the formation of neovasculature. The accompanying table (Table 1) provides specific examples of these ECM constituents and their reported roles in cancer. This table is by no means an exhaustive list of ECM members or function; interested readers should see cited reviews and other excellent reviews not cited due to space limitations.
Future studies should focus on improving current methods as well as developing new approaches to target these molecular components of the tumor microenvironment.

\section{References}

1. Frantz C, Stewart KM, Weaver VM (2010) The extracellular matrix at a glance. J Cell Sci 123: 4195-4200.

2. Lu P, Weaver VM, Werb Z (2012) The extracellular matrix: a dynamic niche in cancer progression. J Cell Biol 196: 395-406.

3. Hynes RO, Naba A (2012) Overview of the matrisome--an inventory of extracellular matrix constituents and functions. Cold Spring Harb Perspect Bio 4: a004903.

4. Kalluri R (2003) Basement membranes: structure, assembly and role in tumour angiogenesis. Nat Rev Cancer 3: 422-433.

5. Couchman JR, Pataki CA (2012) An introduction to proteoglycans and their localization. J Histochem Cytochem 60: 885-897.

6. Levy-Adam F, Feld S, Suss-Toby E, Vlodavsky I, Ilan N (2008) Heparanase facilitates cell adhesion and spreading by clustering of cell surface heparan sulfate proteoglycans. PLoS One 3: e2319.

7. Sanderson RD (2001) Heparan sulfate proteoglycans in invasion and metastasis. Semin Cell Dev Biol 12: 89-98.

8. Roy M, Marchetti D (2009) Cell surface heparan sulfate released by heparanase promotes melanoma cell migration and angiogenesis. J Cell Biochem 106: 200 209.

9. Barash U, Cohen-Kaplan V, Dowek I, Sanderson RD, Ilan N, et al. (2010) Proteoglycans in health and disease: new concepts for heparanase function in tumor progression and metastasis. FEBS J 277: 3890-3903.

10. Rosen SD, Lemjabbar-Alaoui H (2010) Sulf-2: an extracellular modulator of cell signaling and a cancer target candidate. Expert Opin Ther Targets 14: 935-949.

11. Hur K, Han TS, Jung EJ, Yu J, Lee HJ, et al. (2012) Up-regulated expression of sulfatases (SULF1 and SULF2) as prognostic and metastasis predictive markers in human gastric cancer. J Pathol 228: 88-98.

12. Egeblad M, Rasch MG, Weaver VM (2010) Dynamic interplay between the collagen scaffold and tumor evolution. Curr Opin Cell Biol 22: 697-706.

13. Levental KR, Yu H, Kass L, Lakins JN, Egeblad M, et al. (2009) Matrix crosslinking forces tumor progression by enhancing integrin signaling. Cel 139: 891-906.

14. Xiao Q, Ge G (2012) Lysyl oxidase, extracellular matrix remodeling and cancer metastasis. Cancer Microenviron 5: 261-273.

15. Akiri G, Sabo E, Dafni H, Vadasz Z, Kartvelishvily Y, et al. (2003) Lysyl oxidaserelated protein-1 promotes tumor fibrosis and tumor progression in vivo. Cancer Res 63: 1657-1666. 
Citation: Weber CE, Driver J, Franzen CA, Mascarenhas JB, Mi Z, et al. (2013) The Constituents and Potential Targets of the Extracellular Matrix: Implications for Carcinogenesis and Cancer Treatment. J Carcinogene Mutagene S13: 006. doi:10.4172/2157-2518.S13-006

Page 6 of 7

16. Lee HO, Mullins SR, Franco-Barraza J, Valianou M, Cukierman E, et al. (2011) FAP-overexpressing fibroblasts produce an extracellular matrix that enhances invasive velocity and directionality of pancreatic cancer cells. BMC Cancer 11: 245

17. Velling T, Risteli J, Wennerberg K, Mosher DF, Johansson S (2002) Polymerization of type I and III collagens is dependent on fibronectin and enhanced by integrins alpha 11 beta 1 and alpha 2beta 1 . J Biol Chem 277 37377-37381.

18. Klotzsch E, Smith ML, Kubow KE, Muntwyler S, Little WC, et al. (2009) Fibronectin forms the most extensible biological fibers displaying switchable force-exposed cryptic binding sites. Proc Natl Acad Sci U S A 106: 1826718272.

19. Campbell ID, Humphries MJ (2011) Integrin structure, activation, and interactions. Cold Spring Harb Perspect Biol 3.

20. DeMali KA, Wennerberg K, Burridge K (2003) Integrin signaling to the actin cytoskeleton. Curr Opin Cell Biol 15: 572-582.

21. Rathinam R, Alahari SK (2010) Important role of integrins in the cancer biology. Cancer Metastasis Rev 29: 223-237.

22. Zaidel-Bar R, Itzkovitz S, Ma'ayan A, lyengar R, Geiger B (2007) Functional atlas of the integrin adhesome. Nat Cell Biol 9: 858-867.

23. Goodman SL, Picard M (2012) Integrins as therapeutic targets. Trends Pharmacol Sci 33: 405-412.

24. Desgrosellier JS, Cheresh DA (2010) Integrins in cancer: biological implications and therapeutic opportunities. Nat Rev Cancer 10: 9-22.

25. Yang N, Mosher R, Seo S, Beebe D, Friedl A (2011) Syndecan-1 in breast cancer stroma fibroblasts regulates extracellular matrix fiber organization and carcinoma cell motility. Am J Pathol 178: 325-335.

26. Teng YH, Aquino RS, Park PW (2012) Molecular functions of syndecan-1 in disease. Matrix Biol 31: 3-16.

27. Purushothaman A, Chen L, Yang Y, Sanderson RD (2008) Heparanase stimulation of protease expression implicates it as a master regulator of the aggressive tumor phenotype in myeloma. J Biol Chem 283: 32628-32636.

28. Endo K, Takino T, Miyamori H, Kinsen H, Yoshizaki T, et al. (2003) Cleavage of syndecan-1 by membrane type matrix metalloproteinase- 1 stimulates cell migration. J Biol Chem 278: 40764-40770.

29. Mahtouk K, Hose D, Raynaud P, Hundemer M, Jourdan M, et al. (2007) Heparanase influences expression and shedding of syndecan-1, and its expression by the bone marrow environment is a bad prognostic factor in multiple myeloma. Blood 109: 4914-4923.

30. Seidel C, Sundan A, Hjorth M, Turesson I, Dahl IM, et al. (2000) Serum syndecan-1: a new independent prognostic marker in multiple myeloma. Blood 95: $388-392$

31. Bourboulia D, Stetler-Stevenson WG (2010) Matrix metalloproteinases (MMPs) and tissue inhibitors of metalloproteinases (TIMPs): Positive and negative regulators in tumor cell adhesion. Semin Cancer Biol 20: 161-168.

32. Coussens LM, Fingleton B, Matrisian LM (2002) Matrix metalloproteinase inhibitors and cancer: trials and tribulations. Science 295: 2387-2392.

33. Page-McCaw A, Ewald AJ, Werb Z (2007) Matrix metalloproteinases and the regulation of tissue remodelling. Nat Rev Mol Cell Biol 8: 221-233.

34. Buchheit CL, Rayavarapu RR, Schafer ZT (2012) The regulation of cancer cell death and metabolism by extracellular matrix attachment. Semin Cell Dev Biol 23: 402-411.

35. Yu Q, Stamenkovic I (2000) Cell surface-localized matrix metalloproteinase-9 proteolytically activates TGF-beta and promotes tumor invasion and angiogenesis. Genes Dev 14: 163-176.

36. Bergers G, Brekken R, McMahon G, Vu TH, Itoh T, et al. (2000) Matrix metalloproteinase- 9 triggers the angiogenic switch during carcinogenesis. Nat Cell Biol 2: 737-744.

37. Xu J, Rodriguez D, Petitclerc E, Kim JJ, Hangai M, et al. (2001) Proteolytic exposure of a cryptic site within collagen type IV is required for angiogenesis and tumor growth in vivo. J Cell Biol 154: 1069-1079.

38. Pozzi A, Zent R (2009) Regulation of endothelial cell functions by basement membrane- and arachidonic acid-derived products. Wiley Interdiscip Rev Syst Biol Med 1: 254-272.
39. Kalluri R, Weinberg RA (2009) The basics of epithelial-mesenchymal transition $\mathrm{J}$ Clin Invest 119: 1420-1428

40. Moustakas A, Heldin $\mathrm{CH}$ (2012) Induction of epithelial-mesenchymal transition by transforming growth factor $\hat{I}^{2}$. Semin Cancer Biol 22: 446-454.

41. Strobl-Mazzulla PH, Bronner ME (2012) Epithelial to mesenchymal transition: new and old insights from the classical neural crest model. Semin Cancer Biol 22: 411-416.

42. Kajita M, Itoh Y, Chiba T, Mori H, Okada A, et al. (2001) Membrane-type 1 matrix metalloproteinase cleaves CD44 and promotes cell migration. J Cell Biol 153: 893-904

43. Masson D, Denis MG, Denis M, Blanchard D, Loirat MJ, et al. (1999) Soluble CD44: quantification and molecular repartition in plasma of patients with colorectal cancer. Br J Cancer 80: 1995-2000.

44. Hernandez-Barrantes S, Toth M, Bernardo MM, Yurkova M, Gervasi DC, et al. (2000) Binding of active (57 kDa) membrane type 1-matrix metalloproteinase (MT1-MMP) to tissue inhibitor of metalloproteinase (TIMP)-2 regulates MT1 MMP processing and pro-MMP-2 activation. J Biol Chem 275: 12080-12089.

45. Wang Z, Juttermann R, Soloway PD (2000) TIMP-2 is required for efficient activation of proMMP-2 in vivo. J Biol Chem 275: 26411-26415.

46. Grogan K. Merck KGaA's cilengitide fails in Phase III. PharmaTimes February 26, 2013.

47. Kim KB, Prieto V Joseph RW, Diwan AH, Gallick GE, et al. (2012) A randomized phase II study of cilengitide (EMD 121974) in patients with metastatic melanoma. Melanoma Res 22: 294-301.

48. Vermorken JB, Guigay J, Mesia R, Trigo JM, Keilholz U, et al. (2011) Phase I/I trial of cilengitide with cetuximab, cisplatin and 5-fluorouracil in recurrent and/o metastatic squamous cell cancer of the head and neck: findings of the phase part. Br J Cancer 104: 1691-1696.

49. Alva A, Slovin S, Daignault S, Carducci M, Dipaola R, et al. (2012) Phase I study of cilengitide (EMD 121974, NSC 707544) in patients with non-metastatic castration resistant prostate cancer, $\mathrm{NCl}-6735$. A study by the DOD/PCF prostate cancer clinical trials consortium. Invest New Drugs 30: 749-757.

50. Friess H, Langrehr JM, Oettle H, Raedle J, Niedergethmann M, et al. (2006) A randomized multi-center phase II trial of the angiogenesis inhibitor Cilengitide (EMD 121974) and gemcitabine compared with gemcitabine alone in advanced unresectable pancreatic cancer. BMC Cancer 6: 285

51. Hersey P, Sosman J, O'Day S, Richards J, Bedikian A, et al. (2010) A randomized phase 2 study of etaracizumab, a monoclonal antibody against integrin alpha(v)beta(3), + or - dacarbazine in patients with stage IV metastatic melanoma. Cancer 116: 1526-1534

52. Bell-McGuinn KM, Matthews CM, Ho SN, Barve M, Gilbert L, et al. (2011) A phase II, single-arm study of the anti-Î $\pm 5 \hat{I}^{2} 1$ integrin antibody volociximab as monotherapy in patients with platinum-resistant advanced epithelial ovarian or primary peritoneal cancer. Gynecol Oncol 121: 273-279.

53. Cianfrocca ME, Kimmel KA, Gallo J, Cardoso T, Brown MM, et al. (2006) Phase 1 trial of the antiangiogenic peptide ATN-161 (Ac-PHSCN-NH(2)), a beta integrin antagonist, in patients with solid tumours. Br J Cancer 94: 1621-1626.

54. O'Day S, Pavlick A, Loquai C, Lawson D, Gutzmer R, et al. (2011) A randomised, phase II study of intetumumab, an anti-Îtv-integrin mAb, alone and with dacarbazine in stage IV melanoma. Br J Cancer 105: 346-352

55. Heidenreich A, Rawal SK, Szkarlat K, Bogdanova N, Dirix L, et al. (2013) A randomized, double-blind, multicenter, phase 2 study of a human monoclonal antibody to human alphanu integrins (intetumumab) in combination with docetaxel and prednisone for the first-line treatment of patients with metastatic castration-resistant prostate cancer. Ann Oncol 24: 329-336.

56. Miller K, Hussain M, Mordenti G, Lannert H (2012) A phase II study (PERSEUS) of two doses of EMD 525797 (DI17E6) in patients with asymptomatic or mildly symptomatic metastatic castrate resistant prostate cancer. European Society for Medical Oncology 2012 Abstract 1252.

57. Carter A (2010) Integrins as target: first phase III trial launches, but questions remain. J Natl Cancer Inst 102: 675-677.

58. Bissett D, O’Byrne KJ, von Pawel J, Gatzemeier U, Price A, et al. (2005) Phase III study of matrix metalloproteinase inhibitor prinomastat in non-small-cell lung cancer. J Clin Oncol 23: 842-849.

59. Hirte H, Vergote IB, Jeffrey JR, Grimshaw RN, Coppieters S, et al. (2006) 
Citation: Weber CE, Driver J, Franzen CA, Mascarenhas JB, Mi Z, et al. (2013) The Constituents and Potential Targets of the Extracellular Matrix: Implications for Carcinogenesis and Cancer Treatment. J Carcinogene Mutagene S13: 006. doi:10.4172/2157-2518.S13-006

A phase III randomized trial of BAY 12-9566 (tanomastat) as maintenance therapy in patients with advanced ovarian cancer responsive to primary surgery and paclitaxel/platinum containing chemotherapy: a National Cancer Institute of Canada Clinical Trials Group Study. Gynecol Oncol 102: 300-308.

60. Sparano JA, Bernardo P, Stephenson P, Gradishar WJ, Ingle JN, et al. (2004) Randomized phase III trial of marimastat versus placebo in patients with metastatic breast cancer who have responding or stable disease after first-line chemotherapy: Eastern Cooperative Oncology Group trial E2196. J Clin Oncol 22: $4683-4690$

61. Whatcott CJ, Han H, Posner RG, Hostetter G, Von Hoff DD (2011) Targeting the tumor microenvironment in cancer: why hyaluronidase deserves a second look. Cancer Discov 1: 291-296.

62. Lee J, Fassnacht M, Nair S, Boczkowski D, Gilboa E (2005) Tumor immunotherapy targeting fibroblast activation protein, a product expressed in tumor-associated fibroblasts. Cancer Res 65: 11156-11163.

63. Wen Y, Wang CT, Ma TT, Li ZY, Zhou LN, et al. (2010) Immunotherapy targeting fibroblast activation protein inhibits tumor growth and increases survival in a murine colon cancer model. Cancer Sci 101: 2325-2332.

64. Olive KP, Jacobetz MA, Davidson CJ, Gopinathan A, McIntyre D, et al. (2009) Inhibition of Hedgehog signaling enhances delivery of chemotherapy in a mouse model of pancreatic cancer. Science 324: 1457-1461.

65. Eikenes L, Bruland ØS, Brekken C, Davies Cde L (2004) Collagenase increases the transcapillary pressure gradient and improves the uptake and distribution of monoclonal antibodies in human osteosarcoma xenografts. Cancer Res 64 4768-4773.

66. Brekken C, Hjelstuen MH, Bruland ØS, de Lange Davies C (2000) Hyaluronidase-induced periodic modulation of the interstitial fluid pressure increases selective antibody uptake in human osteosarcoma xenografts. Anticancer Res 20: 3513-3519.

67. Brekken C, Bruland ØS, de Lange Davies C (2000) Interstitial fluid pressure in human osteosarcoma xenografts: significance of implantation site and the response to intratumoral injection of hyaluronidase. Anticancer Res 20: 35033512.

68. Provenzano PP, Cuevas C, Chang AE, Goel VK, Von Hoff DD, et al (2012) Enzymatic targeting of the stroma ablates physical barriers to treatment of pancreatic ductal adenocarcinoma. Cancer Cell 21: 418-429.

69. Ismael G, Hegg R, Muehlbauer S, Heinzmann D, Lum B, et al. (2012) Subcutaneous versus intravenous administration of (neo)adjuvant trastuzumab in patients with HER2-positive, clinical stage I-III breast cancer (HannaH study): a phase 3, open-label, multicentre, randomised trial. Lancet Oncol 13: 869-878.

70. Hamizi S, Freyer G, Bakrin N, Henin E, Mohtaram A, et al. (2013) Subcutaneous trastuzumab: development of a new formulation for treatment of HER2-positive early breast cancer. Onco Targets Ther 6: 89-94.
This article was originally published in a special issue, Cell-Extracellula Matrix Interactions in Carcinogenesis handled by Editor(s). Dr. Claudio Luparello, University of Palermo, Italy 